\title{
HOXA11-AS1 promotes PD-L1 mediated immune escape and metastasis of hypopharyngeal carcinoma by facilitating the association of PTBP1 with FOSL1
}

\section{Zheng Zhou}

Third Xiangya Hospital

Qian Liu

Third Xiangya Hospital

Gehou Zhang

Third Xiangya Hospital

Diab Mohammed

Xiangya Hospital Central South University

\section{Sani Amadou}

Maradi Reference Hospital, Maradi

Guolin Tan

Third Xiangya Hospital

Xiaowei Zhang ( $\square$ xiaowei-zhang@csu.edu.cn )

Third Xiangya Hospital

\section{Research Article}

Keywords: hypopharyngeal squamous cell carcinoma, HOXA11-AS1, PD-L1, FOSL1, PTBP1, immune escape

Posted Date: March 28th, 2022

DOI: https://doi.org/10.21203/rs.3.rs-1287515/v2

License: (c) (1) This work is licensed under a Creative Commons Attribution 4.0 International License. Read Full License 


\section{Abstract \\ Background}

The metastatic characteristics of hypopharyngeal squamous cell carcinoma (HSCC) led to many diagnostic and therapeutic challenges. The identification of functional long non-coding RNAs (IncRNAs) would contribute to investigate effective strategies for the diagnosis and treatment of HSCC.

\section{Methods}

RT-qPCR, western blot, immunohistochemistry and immunofluorescence assay were used to detect the related gene expression. Flow cytometry was used to measure the percentage of $\mathrm{CD} 8^{+}$and $\mathrm{CD} 4^{+} \mathrm{T}$ cells. CCK-8 and transwell assays were performed to analyze the functional role of HOXA11-AS1. The targeted relationship of FOSL1/PD-L1 promoter was measured by ChIP and dual-luciferase reporter assays. RNA pull-down and RIP assays were used to measure the interaction between HOXA11-AS1, FOSL1 and PTBP1. Tumor xenograft study was used to analyze the function of HOXA11-AS1 in vivo.

\section{Results}

HOXA11-AS1, PD-L1 and FOSL1 were up-regulated in HSCC, and HOXA11-AS1 was positively correlated with PD-L1. HOXA11-AS1 knockdown upregulated CD8 + T cells and the concentration of IFN-y, while decreased the proliferation, migration and invasion of HSCC cells. FOSL1 bound to PD-L1 promoter and increased its expression. HOXA11-AS1 enhanced the stability of FOSL1 mRNA by binding to PTBP1.

Overexpression of HOXA11-AS1 or PTBP1 increased the expression of FOSL1 and PD-L1. Knockdown of PD-L1 arrested the inhibiting function of HOXA11-AS1 overexpression on CD8 ${ }^{+} \mathrm{T}$ cells content. Knockdown of HOXA11-AS1 inhibited the immune escape and metastasis through regulating PD-L1 by down-regulating FOSL1 in vivo.

\section{Conclusion}

HOXA11-AS1 promoted PD-L1 expression through up-regulating FOSL1 levels by PTBP1, thereby facilitating the immune escape, proliferation and metastasis of HSCC cells.

\section{Introduction}

Hypopharyngeal squamous cell carcinoma (HSCC) is one of the most frustrating and aggressive malignancies in the hypopharynx, accounting for $3-5 \%$ of all head-and-neck tumors(1). Patients with HSCC are often advanced at the time of diagnosis, which caused by the delayed diagnosis due to the absence of initial symptoms(2). Coupled with the metastatic characteristics such as submucosal spread 
and cervical lymph node metastasis, and the high incidence of recurrent, seriously affect the five-year survival rate-less than $40 \%$ in stage III and IV patients, which have no encouraging improvement with contemporary treatments(3-5). Therefore, it is very important to explore and discover molecular targets for HSCC diagnosis and treatment.

Programmed death-ligand 1 (PD-L1, also named CD274 or B7-H1) act as a coinhibitory molecule in the development of cancers, which is expressed on the surface of $\mathrm{T}$ lymphocytes and facilitates the immune escape $(6,7)$. Growing evidences proved that the up-regulation of PD-L1 was involved in the aggressive behavior of tumors and is closely related to poor prognosis(8). For example, Wintterle et al. suggested that PD-L1 modulated CD4 + and CD8 + T cell levels by increasing cytokine concentration, such as IFN- $y$, thus showing immunomodulatory function in glioma cells(9). Similarly, the high expression of PD-L1 facilitated HSCC cells to escape recognition of the host immune system and accelerated the tumor metastasis $(10,11)$, indicating that the inhibition of PD-L1 might has positive effect for HSCC treatment. However, the specific function mechanism of PD-L1 needs more investigation.

The transcription factor FOSL1 is widely reported to play a role in promoting metastasis in a variety of cancers, such as breast cancer(12), clear cell renal cell carcinoma(13) and bladder cancer(14). Although the role of FOSL1 in HSCC remained largely unknown, TCGA database predicted high expression of FOSL1 in head-and-neck cancer, and microarray assay also indicated that FOSL1 was overexpressed in HSCC. More importantly, JASPAR prediction indicated binding sequence between FOSL1 and PD-L1 promoter. Therefore, we suspected that the interaction between them might be a potential breakthrough to explore the immune escape and metastasis process of HSCC. Increasing studies have shown that the abnormal expression of long non-coding RNAs (IncRNAs) play roles as tumor promoters or suppressors, which provide new perspectives for exploring the pathogenesis of cancers $(15,16)$. In the study of HSCC, IncRNA UCA1 had been reported highly expressed in HSCC, and act as a pro-metastatic gene and a tumor promoter in vitro(17). This study was calculated to elucidate the molecular mechanism of homeobox A11 antisense RNA1 (HOXA11-AS1) in HSCC, which was also poorly studied in other tumors. Our microarray analysis indicated that the expression HOXA11-AS1 was up-regulated in HSCC. Remarkably, Starbase predicted binding sequences between HOXA11-AS1 or FOSL1 and polypyrimidine tract binding protein 1 (PTBP1), which belongs to the family of heterogeneous nuclear ribonucleoproteins, more importantly, promoted tumor progression through regulating the selective splicing and stability of mRNA(18). We therefore speculated that HOXA11-AS1 promoted the association between PTBP1 and FOSL1, and participated in the metastasis of HSCC.

In this study, we aimed to explore the up-regulation of HOXA11-AS1 to promote the FOSL1 mRNA mediated by PTBP1 and increase the expression of PD-L1, which were conducive to the immune escape and metastasis of HSCC, to provide a new feasible target for the diagnosis and treatment of HSCC.

\section{Materials And Methods}

\section{Patients}


40 samples of HSCC and matched adjacent normal specimens were obtained from patients with HSCC who did not receive radiotherapy or chemotherapy at Third Xiangya hospital of Central South University from January 2016 to June 2020 . All samples were stored in $-80^{\circ} \mathrm{C}$ until analysis. The study was

approved by the Ethics Committee of Third Xiangya hospital of Central South University, and all subjects signed written informed consent.

\section{Cell culture and transfection}

Human pharyngeal carcinoma cells (FaDu and Detroit 562) and human nasopharyngeal epithelial cells (NP69), were obtained from the American Type Culture Collection (Manassas, VA, USA), and then cultured in Dulbecco's modified Eagle medium (Thermo Fisher Scientific, Inc. Waltham, MA, USA) supplemented with $10 \%$ fetal calf serum (Invitrogen, NY, CA, USA), $100 \mathrm{U} / \mathrm{mL}$ penicillin and $100 \mu \mathrm{g} / \mathrm{mL}$ streptomycin (Invitrogen) at $37^{\circ} \mathrm{C}$ in a humidified incubator with $5 \% \mathrm{CO}_{2}$.

The short hairpin RNA (shRNA) knocking down HOXA11-AS1, PD-L1 and PTBP1, the lentiviral plasmids overexpressing HOXA11-AS1 and PTBP1 were synthesized by RiboBio (Guangzhou, China). All plasmids and the combination were transduced into FaDu or Detroit 562 cells using Lipofectamine 3000 (Invitrogen).

\section{RNA extraction and quantitative real-time PCR (qRT-PCR) assay}

Total RNA from tissues or cells was extracted by TRIzol reagent (Invitrogen), and the concentration was measured using NanoDrop Spectrophotometer (Thermo). RevertAid First Strand cDNA Synthesis kit (Thermo) was applied for reverse transcription. An ABI qRT-PCR 7900 system (Thermo) was employed to perform PCR reaction. Gene expression was normalized to GAPDH. Primers were synthesized by RiboBio and showed as follow: 5 -CGGCTAACAAGGAGATTTGG-3 (sense) and 5 -AGGCTCAGGGATGGTAGTCC3 (antisense) for HOXA11-AS, 5 -: GTGGCATCCAAGATACAAACTCAA-3 (sense) and 5 TCCTTCCTCTTGTCACGCTCA-3 (antisense) for PD-L1, 5 - CCGAAGAAAGGAGCTGACAGAC -3 (sense) and 5 - -3 (antisense) for FOSL1, 5 - CTCAAGGCGTTCCTTCTGCTTC -3 (sense) and 5 GGAGGAGTGGGTGTCGCTGT-3 (antisense) for GAPDH.

\section{Western blot}

Protein extraction was carried out with lysis buffer (Beyotime Institute of Biotechnology, Haimen, China). Then proteins were quantified by an acid-based Protein Assay Kit (Thermo), followed by separate with $10 \%$ SDS-PAGE by electrophoresis and transfer to polyvinylidene difluoride membranes (Millipore, Bedford, MA, USA). Afterwards, $5 \%$ skim milk ( $2 \mathrm{~h}$ ) was used to block the non-specific binding sites of the membranes. The membranes were subsequently incubated with indicated primary antibodies overnight against PD-L1 (1:1000, ab213524, Abcam, Cambridge, UK), FOSL1 (1:1000, ab252421, Abcam), PTBP1 (1:5000, ab134950, Abcam), followed by incubate with horseradish peroxidase-conjugated secondary antibodies. Washed with PBS for three times and the signals were visualized by ECL reagent (Millipore). 
Protein expression was then analyzed by ImageJ software 6.0 (National Institutes of Health, USA). GAPDH and $\beta$-actin acted as an internal control.

\section{Immunohistochemistry (IHC)}

Paraffin sections of HSCC tumor tissues were deparaffinized and hydrated for incubating with primary antibodies (anti-PD-L1 (1:500, ab228415, Abcam) overnight, followed by incubate with secondary antibody for $30 \mathrm{~min}$. After developing by Diaminobenzidine substrate, sections were counterstained with hematoxylin. Images were visualized using a Nikon ECLIPSE Ti microscope system and processed with Nikon software.

\section{CCK-8 assay}

Cells were seeded into culture plate and incubate for indicated time. Then $10 \mu \mathrm{L}$ CCK-8 solution was added for treating cells. Absorbance was detected at $490 \mathrm{~nm}$ by a Multiskan ${ }^{\mathrm{TM}} \mathrm{GO}$ microplate spectrophotometer (Thermo).

\section{Colony formation assay}

After trypsinizing, cells $\left(1 \times 10^{5}\right)$ were grown in 6-well plates for 2 weeks under a humidified atmosphere. The colonies were fixed with $4 \%$ paraformaldehyde and then stained with $0.1 \%$ crystal violet. A microscope was used to calculate the number of colonies.

\section{Wound healing assay}

Briefly, treated cells plated in 6-well plates were cultured at $37^{\circ} \mathrm{C}$ until $100 \%$ confluence, followed by scratching a straight wound on the surface of cell layer using a sterile pipette. Next, debris on the cell surface was removed by washing with PBS twice, and incubated cells for $24 \mathrm{~h}$. Took a photograph of migrating cells after $0 \mathrm{~h}$ and $24 \mathrm{~h}$ of scratching by a microscope.

\section{Transwell assay}

Cell invasion capability was measured by Transwell chambers with an 8- $\mu$ m pore size (Corning, Tewksbury, MA, USA) coated with Matrigel. Briefly, $1 \times 10^{5}$ cells in $100 \mu \mathrm{L}$ of serum-free RPMI 1640 medium were placed into the upper chamber. $500 \mu \mathrm{L}$ medium containing 10\% FBS was added in the lower chamber. Cells were dyed in $0.5 \%$ crystal violet after $48 \mathrm{~h}$ culture at $37^{\circ} \mathrm{C}$ with $5 \% \mathrm{CO}_{2}$. The cells remaining in the upper chamber were removed. Following washing with PBS and drying, images were taken under a microscope (Olympus, Japan).

\section{Co-culture of human PBMC and HSCC cells}

FaDu and Detroit 562 cells with or without PD-L1 antibody were selected for co-culture experiments. Human peripheral blood mononuclear cell (PBMC) was isolated by Ficoll-Hypaque gradient 
centrifugation. Treated cells were co-cultured with PBMC at a ratio of $1: 4$ for at least $72 \mathrm{~h}$ for further analyzed.

\section{Flow cytometry}

Co-cultured cells were staining with following antibodies: anti-CD3 (317320, BioLegend, San Diego, CA, USA), anti-CD4 (357402, BioLegend) and anti-CD8 (344702, BioLegend), then assessed using a BD FACSAria III flow cytometer (BD Biosciences, San Jose, CA, USA), and data were analyzed with FlowJo V10 software (TreeStar Inc., Ashland, OR, USA).

\section{Enzyme-linked immunosorbent assay (ELISA)}

Cell-free supernatant from co-cultured cells was assessed for interferon- $\gamma$ (IFN- $\gamma$ ) concentration by human ELISA kit according to the manufacturer's instructions (Multisciences, Hangzhou, China).

\section{Immunofluorescence assay}

The transfected cells were fixed with $4 \%$ formaldehyde for $20 \mathrm{~min}$ at room temperature. After $1 \mathrm{~h}$ incubating with 5\% Tris buffered saline with Tween-20 (pH8.3) diluted non-fat dry milk, cells were incubated with the primary antibody PD-L1 (1:200, ab228415, Abcam) overnight at $4^{\circ} \mathrm{C}$ and then the corresponding secondary antibody at $37^{\circ} \mathrm{C}$ for $1 \mathrm{~h}$. Next, DAPI was used to stain cell nuclear, and a confocal laser scanning microscope was used to analyze the immunofluorescence images.

\section{Fractionation of nuclear/cytoplasmic RNA}

Nuclear/cytoplasmic fractionation was performed by PARIS Kit (Life Technologies, MA, USA). Briefly, cells were washed with PBS, followed by centrifuging and lysing in $1 \mathrm{ml}$ of lysis buffer. Cytoplasmic RNA was subsequently obtained from supernatant, and the remaining nuclear pellet was washed three times with hypotonic lysis buffer to extracted nuclear RNA.

\section{The chromatin immunoprecipitation (ChIP) assay}

The two binding sites (BS1: TGTGTCAT; BS2: AAATCACTGAGCAGCAAG

CTGA) between FOSL1 and PD-L1 were predicted by JASPAR website (http://jaspar.genereg.net/). The kit from Millipore (Bedford, MA, USA) was used to perform ChIP assay. Briefly, cells were cross-linked with $1 \%$ formaldehyde for $10 \mathrm{~min}$, then lysed and sonicated to obtain chromatin fragments of 500 average size. $1 \%$ of supernatant was collected to serve as an input control. The chromatin diluted by ChIP solution was immunoprecipitated with DNMT3A antibody or $\operatorname{lgG}$ at $4^{\circ} \mathrm{C}$ overnight with rotation. After reversing the cross-links, the immune complexes were purified and analyzed by PCR.

\section{Dual-luciferase reporter assay}


The PD-L1 promoter fragment containing the two binding sites (wild type BS1 and mutant BS2, or mutant BS1 and wild type BS2) was subcloned into pGL3-basic vector (Promega, USA), thereby obtaining recombinant plasmids: mut-BS2 and mut-BS1. The FOSL1 CDNA was PCR amplified and inserted into the pcDNA3.1 vector (Promega), empty vector (EV) was used as control. The 293T cells were co-transfected with pGL3 luciferase construct (mutant BS1 or mutant BS2) and FOSL1 expression or empty plasmids. Luciferase of indicated transfected cells was estimated using Dual-Luciferase Reporter Assay System (Promega, Madis on, WI, USA) after $48 \mathrm{~h}$ of transfection.

\section{mRNA stability analysis}

HOXA11-AS1 or PTBP1 silenced cells were treated with $5 \mu \mathrm{g} / \mathrm{mL}$ actinomycin D (MedChemExpress, NJ, USA). Total RNA was extracted at indicated time, and then HOXA11-AS1 or FOSL1 mRNA was measured using RT-qPCR and normalized to GAPDH.

\section{RNA pull-down}

Biotin-labeled HOXA11-AS1 and FOSL1 mRNA or their antisense RNA were transcribed with the Biotin RNA Labeling Mix and T7/SP6 RNA polymerase (Roche Diagnostics, Indianapolis, IN, USA), then treated with RNase-free DNase I (Roche). After purifying with the RNeasy Mini Kit (Roche), biotin-labeled RNAs were mixed with extracted FaDu and Detroit 562 cell nuclear proteins and then incubated with streptavidin agarose beads at room temperature for $1 \mathrm{~h}$. The protein bands were visualized by silver staining followed by western blot.

\section{RNA immunoprecipitation (RIP) assay}

RIP assay was conducted using a Magna RIP ${ }^{T M}$ RNA-Binding Protein Immunoprecipitation Kit (Millipore). Cells were lysed with RIP lysis buffer and then immune-precipitated with immunoglobulin $\mathrm{G}$ antibody (anti-lgG) and argonaute 2 antibody (anti-Ago2) coated on magnetic beads overnight, followed by wash with PBS. Part of the cell was used as the negative control, named Input. The co-precipitated RNA was extracted using TRIzol ${ }^{\mathrm{TM}}$, and RT-qPCR was then used to analyze the purified RNA.

\section{In vivo xenograft experiments}

Forty-eight NOD-SCID mice (male, 18-22 g, 6 weeks) used in this study were purchased from Hunan slake Jingda Co., Ltd (Changsha, China). FaDu or Detroit 562 cells stably expressing shHOXA11-AS1-1, shHOXA11-AS1-2 or shNC were injected subcutaneously into the right flank of mice $(n=4)$ to perform tumorigenesis assay. Next, each xenograft tumor model was assigned into 2 groups $(n=4)$, and then was injected intraperitoneally with PBS or PBMC for killing xenograft effectively. Tumor volumes were calculated every 5 days by the equation: volume $\left(\mathrm{mm}^{3}\right)=0.5 \times(\text { Width })^{2} \times($ Length). At 30 days after injection, mice were euthanized to obtain tumors. The sections of tumor tissue were stained by hematoxylin and eosin (HE) or ki67 to analyze the pathology or proliferation of tumor tissue. IHC staining 
was performed to measure PD-L1 levels. Tumor regression rate $=$ (tumor volume in NOD-SCID mice after PBMC treatment /tumor volume in NOD-SCID mice after PBS treatment) $\times 100 \%$.

For metastasis assay, FaDu or Detroit 562 cells stably expressed shHOXA11-AS1-1, shHOXA11-AS1-2 and shNC were injected into mice through tail vein $(n=4)$. After 4 weeks with injection, mice were sacrificed, and lung lobes were removed and then harvested and placed in 10\% neutral-buffered formalin fixative overnight and embedded in paraffin. Then, sections were stained by HE for measure the pathology, as well as observed and counted the metastasis foci within mice lungs and visible lung metastatic nodules using a light microscope (Olympus Cororation, Tokyo, Japan). All animal experiments were approved by the Animal Ethics Committee of Third Xiangya hospital of Central South University.

\section{Statistical Analysis}

Data were given as the mean and standard deviation (SD). Students' t-test was used to compare the difference between two groups for continuous variables. One-way analysis of variance (ANOVA) followed by Tukey post hoc test was used for multiple comparisons. All the analyses were performed using GraphPad Prism 6 (GraphPad Software, Inc.) P $<0.05$ was considered statistically significant.

\section{Results}

HOXA11-AS1 and PD-L1 were highly expressed in HSCC, and HOXA11-AS1 was positively correlated with PD-L1.

In this study, HOXA11-AS1 and PD-L1 levels in HSCC and normal tissues were evaluated. As shown in Fig. 1A and 1B, HOXA11-AS1 and PD-L1 were highly expressed in HSCC tissues. HSCC patients with lower levels of HOXA11-AS1 or PD-L1 had increased survival rate compared with those with higher levels of HOXA11-AS1 or PD-L1 (Fig. 1C and 1D). Then, the correlation between HOXA11-AS1 and PD-L1 was analyzed, and the positive correlation was confirmed (Fig. 1E). To further verify the up-regulation of PD-L1 in HSCC tissues, IHC staining was adopted to measure PD-L1 levels in tumor tissues. As shown in Fig. 1F, PD-L1 was overexpressed. Moreover, we detected increased expression of HOXA11-AS1 and PD-L1 in HSCC cells (Fig. $1 \mathrm{G}$ and $1 \mathrm{H}$ ).

\section{Knockdown of HOXA11-AS1 suppressed the expression of PD-L1, as well as the immune escape, proliferation and metastasis of HSCC cells.}

To explore the cellular role of HOXA11-AS1, two independent plasmids were used to deplete HOXA11-AS1, which increased specificity and helped to eliminate off-target effects from the use of a single plasmid in FaDu and Detroit 562 cell lines. HOXA11-AS1 was efficiently silenced by transfecting with shHOXA11AS1-1 and shHOXA11-AS1-2 (Fig. 2A). Since HOXA11-AS1 positively associated with PD-L1, we then measured PD-L1 levels after silencing HOXA11-AS1 and observed down-regulation of PD-L1 (Fig. 2B and 2C). The dysregulation of PD-L1, which could regulate the proliferation and cytotoxicity of T cells, had been reported to be a cogent mechanism for potentially immunogenic tumors to escape from host 
immune responses $(8,19)$, thus, HOXA11-AS1 silenced cells were co-cultured with PBMC to evaluate whether the reduction of PD-L1 induced by HOXA11-AS1 knockdown could affect the concentration of $\mathrm{CD} 8^{+}$and $\mathrm{CD} 4^{+} \mathrm{T}$ cells in vitro. As shown in Fig. 2D-E, knockdown of HOXA11-AS1 or anti-PD-L1 treatment could increase $\mathrm{CD} 8^{+} \mathrm{T}$ cell percentages while decrease $\mathrm{CD} 4^{+} \mathrm{T}$ cells in PBMC, as expected, HOXA11-AS1 knockdown and PD-L1 antibody addition together further upregulated $C D 8^{+} \mathrm{T}$ cell contents while downregulated $\mathrm{CD} 4^{+} \mathrm{T}$ cell contents, suggesting the activation of $\mathrm{T}$ lymphocytes. Furthermore, knockdown of HOXA11-AS1 or anti-PD-L1 treatment increased the concentration of IFN- $\gamma$, and silencing of HOXA11-AS1 and the addition of PD-L1 antibody further promoted the secretion of IFN- $\gamma$ by PBMC (Fig. 2F), further supporting our conclusion that HOXA11-AS1 facilitated the immune escape in HSCC cells. Next, we analyzed the effects of HOXA11-AS1 knockdown on the proliferation and metastasis of HSCC cells, and we found that the cell viability and colony formation were inhibited by silencing of HOXA11-AS1 (Fig. 2G and 2H). Similarly, the capability of cell migration and invasion was reduced after knockdown of HOXA11-AS1 (Fig. 2l and 2J). Taken together, HOXA11-AS1 promoted cell proliferation, metastasis and immune escape through regulating PD-L1 in vitro.

\section{FOSL1 was highly expressed in HSCC cells and positively regulated PD-L1 expression through binding to the promoter of PD-L1.}

TCGA database predicted that FOSL1 was significantly expressed in head-and-neck cancers, we had reason to explore the expression and potential pathway of FOSL1 in HSCC cells. FOSL1 was observed to be overexpressed in FaDu and Detroit 562 cells (Fig. 3A). Then FOSL1 was successfully depleted by two plasmids, inducing the down-regulation of PD-L1 in vitro (Fig. 3B), we therefore speculated that there might be a targeting relationship between FOSL1 and PD-L1. We found two binding sites between FOSL1 and PD-L1 promoter, namely BS1 (136 to 157 bp upstream of transcription start site (TSS)) and BS2 (580 to $587 \mathrm{bp}$ upstream of TSS), and the binding sequences were illustrated in Fig. 3C. Subsequently, we found that FOSL1 only bound to BS2 of PD-L1 promoter, not BS1 (Fig. 3D), which was verified in Fig. 3E, compared to mutant BS2, FOSL1 was significantly bound to mutant BS1. These findings demonstrated that FOSL1 transcriptionally regulated PD-L1 levels in HSCC cells.

\section{HOXA11-AS1 enhanced the stability of FOSL1 mRNA through combining with PTBP1.}

Since HOXA11-AS1 and FOSL1 both contributed to the expression of PD-L1, we focused on exploring the regulating role between them. We firstly observed that FOSL1 levels were decreased after silencing of HOXA11-AS1 (Fig. 4A and 4B). To better investigate the function of HOXA11-AS1, we analyzed the specific cellular fractionation and found that HOXA11-AS1 was mainly distributed in the cytoplasm (Fig. 4C), indicating that it might facilitate downstream genes at posttranscriptional level. Therefore, we measured whether the FOSL1 mRNA stability was regulated by HOXA11-AS1. After treating with actinomycin D, we found that HOXA11-AS1 knockdown shortened the half-life of FOSL1 mRNA (Fig. 4D), suggesting that HOXA11-AS1 stabilized FOSL1 mRNA. Subsequently, Starbase predicted that both HOXA11-AS1 and FOSL1 could bind to PTBP1, indicating that PTBP1 might be involved in the regulation of FOSL1 as a specific protein partner of HOXA11-AS1. RNA pull-down and RIP assays were carried out to 
confirm this speculation. As shown in Fig. 4E and 4F, PTBP1 bound to sense of HOXA11-AS1 and FOSL1 mRNA, and the abundance of PTBP1 binding to HOXA11-AS1 and FOSL1 mRNA was much higher than that of IgG group, identifying PTBP1 could act as a binding protein of HOXA11-AS1 and FOSL1. Furthermore, PTBP1 silencing induced the low expression of HOXA11-AS1 and FOSL1 in FaDu and Detroit 562 cells (Fig. 4G), as well as shortened the half-life of HOXA11-AS1 and FOSL1 mRNA (Fig. 4H-I). These finding indicated a targeting relationship of HOXA11-AS1 and PTBP1, and validated the enhancement effect of HOXA11-AS1 on the interaction between PTBP1 and FOSL1 mRNA.

\section{HOXA11-AS1 promoted PD-L1 expression through up-regulating FOSL1 levels by PTBP1, thereby} facilitating cell immune escape, growth and metastasis.

To further investigate the role of HOXA11-AS1/PTBP1/FOSL1 axis on the regulation of PD-L1 and biological function in vitro, we firstly stably overexpressed HOXA11-AS1 or PTBP1 by transfecting lentivirus. The results suggested that overexpression of HOXA11-AS1 or PTBP1 increased FOSL1 and PDL1 levels in FaDu and Detroit 562 cells (Fig. 5A and 5B). Then the shRNA against PTBP1 was used to silence PTBP levels. As expected, PTBP1 knockdown decreased the expression of FOSL1 and PD-L1, more importantly, partially reversed the upregulation of FOSL1 and PD-L1 caused by HOXA11-AS1 overexpression (Fig. 5C). Interestingly, knockdown of PD-L1 was subsequently to exert the same reverse effects on high expression of PD-L1 caused by HOXA11-AS1 overexpression, while the increased FOSL1 levels had no changes (Fig. 5D). These data demonstrated that HOXA11-AS1 regulated PD-L1 through facilitating the association of PTBP1 with FOSL1. Since HOXA11-AS1 promoted PD-L1 levels, proliferation, metastasis and immune escape in HSCC cells, we then investigated the regulating mechanism of PD-L1 in HOXA11-AS1 overexpressed cells. As shown in Fig. 5E, HOXA11-AS1 overexpression decreased $C D 8+T$ cell percentages while increased $C D 4+T$ cell percentages, knockdown of PD-L1 led to the opposite results, and partially reversed the increased of T lymphocytes induced by HOXA11-AS1 overexpression. Moreover, knockdown of PD-L1 increased the concentration of IFN- $y$ that reduced by HOXA11-AS1 overexpression (Fig. 5F), indicating that HOXA11-AS1 facilitated the immune escape in HSCC cells by regulating PD-L1. Similarly, HOXA11-AS1 enhancement facilitated cell proliferation, migration and invasion, while these promoting effects were reversed by PD-L1 knockdown (Fig. 5G-5J). We therefore confirmed the role of HOXA11-AS1/PD-L1/PTBP1/FOSL1 axis in promoting the progression of HSCC in vitro.

\section{Knockdown of HOXA11-AS1 inhibited the immune escape and metastasis through regulating PD-L1 by down-regulating FOSL1 in vivo.}

To assess the anti-tumor effect of HOXA11-AS1 knockdown in vivo, PBMC was firstly injected into NODSCID mice to establish human immune system, then FaDu and Detroit 562 cells stably expressed shNC, shHOXA11-AS1-1 or shHOXA11-AS1-2 were subcutaneously into mice. Then we observed that after silencing HOXA11-AS1 or treating with PBMC, the pathological degree and proliferation of tumor, as well as the expression of PD-L1 and Ki-67 were reduced, while these inhibition effects were further enhanced by knockdown of HOXA11-AS1 and addition of PBMC together (Fig. 6A-B), indicating the effective anti- 
tumor role of HOXA11-AS1 deletion in vivo. The results suggested that tumors were shrank after knockdown of HOXA11-AS1 (Fig. 6C), indicating that the inhibiting effect of T cells on tumor cells could be restored by knockdown of HOXA11-AS1.

Furthermore, we observed the decreased volume and weight of tumor after silencing HOXA11-AS1 (Fig. 6D-6F). Then, PTBP1, FOSL1 and PD-L1 levels in vivo decreased after knockdown of HOXA11-AS1 (Fig. 6G). Moreover, the tumor metastasis capability was also affected by the depletion of HOXA11-AS1. As shown in Fig. 6H-6I, HOXA11-AS1-knockdown impaired the ability of the tumor to metastasis to lung. Taken together, the depletion of HOXA11-AS1 combined with the blocking of PD-L1 showed a more effective function on the inhibition of HSCC progression in vivo.

\section{Discussion}

One of the most difficult challenges during the treatment of HSCC is the invasion and metastasis to adjacent structures due to extensive lymphatic drainage $(20,21)$. Therefore, the investigation of new therapeutic targets has had a high profile in recent years. LncRNAs and their related mechanisms have also been extensively explored, which are involved in many processes of cancer development, such as proliferation, differentiation and metastasis(22-25). In addition, since PD-L1 is often reported to be overexpressed in malignant tumors, mechanically, helps cancer cells evade recognition by the host immune system, PD-L1 has also become the focus of oncologic research(26, 27). Previous studies have reported the immunopathogenic effect and cancer-intrinsic function of PD-L1 in $\operatorname{HSCC}(11,28)$, however, in general, the contributions of PD-L1 in the pathogenesis still need more discussion. In this study, we found that knockdown of HOXA11-AS1 suppressed immune escape and metastasis in HSCC through down-regulating PD-L1 levels by inhibiting the interaction between FOSL1 and PTBP1.

In recent years, the function of IncRNAs in HSCC has attracted increasing attention. For instance, IncRNA PEG10 was highly expressed in HSCC, and overexpression of IncRNA PEG10 facilitated cell proliferation and metastasis in vitro(29). HOXA11-AS was one of the most overexpressed IncRNAs in HSCC and positively associated with lymph node metastasis(30). HOXA11-AS1 was up-regulated in HSCC suggested by microarray analysis, we therefore chose HOXA11-AS1 as the present research object. The high expression of HOXA11-AS1 was measured both in tissues and cells. Functionally, HOXA11-AS1 silencing suppressed cell growth, metastasis, and immune escape of HSCC cells to CD ${ }^{+} \mathrm{T}$ cells, which was a key inducement for tumor progression. Interestingly, we observed that PD-L1 expression was positively correlated with HOXA11-AS1. The regulatory relationship between IncRNAs and PD-L1 had been reported in previous tumor related studies $(31,32)$. For example, SNHG14 induced the inactivation of $\mathrm{CD}^{+} \mathrm{T}$ cells and the promotion of immune escape of diffuse large $\mathrm{B}$ cell lymphoma cell by activating PDL1(33). This is consistent with our findings that the elimination of HOXA11-AS1 combined with PD-L1 antibody more effectively upregulated the content of $\mathrm{CD} 8^{+} \mathrm{T}$ cells in vitro, suggesting an association between HOXA11-AS1 and PD-L1 immune checkpoint at first time in HSCC. 
Exploring the biological targets related to HOXA11-AS1 or PD-L1 can better clarify their molecular mechanisms in HSCC, and further improve the possibility of HOXA11-AS1 in clinical application. Our findings indicated that FOSL1 bound to PD-L1 promoter and positively regulated its expression in vitro. FOSL1 was reported to act as an oncogene in a variety of cancers, and the tumor-intrinsic functions of FOSL1 had been widely investigated(34). For instance, FOSL1 transcription factor had been demonstrated to be a major effector of RAS-ERK1/2 pathway and FOSL1 could increase the metastatic capability of pancreatic cancer through activating epithelial-mesenchymal transition $(35,36)$. Our findings revealed the other possible functions of FOSL1 in tumor, such as the promoting effect on immune escape of tumor cells, by demonstrating an interaction between FOSL1 and PD-L1. PTBP1 is an RNA-binding protein that could shuttle from nucleus to cytoplasm and then perform different functions, and the main functions in cytoplasm include localization, regulation of translation initiation and maintenance of mRNA stability $(37,38)$. In this study, HOXA11-AS1 was observed to mostly distribute in cytoplasm and bound to PTBP1, thus enhanced the stability of FOSL1 mRNA, indicating that HOXA11-AS1 promoted the association of PTBP1 with FOSL1. Previous studies had been suggested the similar function of other IncRNAs. Li et al. indicated that IncRNA ANCR reduced the differentiation ability of human adiposederived mesenchymal stem cells to definitive endoderm through enhancing the interaction between PTBP1 and ID2(39). Functionally, we found that PTBP1 silencing could reverse the up-regulation effect of HOXA11-AS1 overexpression on FOSL1 and PD-L1. More importantly, knockdown of PD-L1 blocked the inhibiting effect on $\mathrm{CD}^{+} \mathrm{T}$ cells and the promoting effect on HSCC cell growth and metastasis induced by HOXA11-AS1 overexpression. Therefore, we concluded that HOXA11-AS1 promoted the immune escape and metastasis of HSCC cells by up-regulating PD-L1 through increasing the interaction between PTBP1 and FOSL1. To a certain extent, the role of this novel pathway had also been confirmed in vivo.

In conclusion, our findings demonstrated the positive regulatory relationship between HOXA11-AS1 and PD-L1, and HOXA11-AS1 knockdown suppressed PD-L1-mediated immune escape and metastasis by reducing the association between PTBP1 and FOSL1 in HSCC, providing a theoretical basis for HOXA11AS1 to be a prognostic marker for HSCC diagnosis and treatment.

\section{Declarations}

\section{Ethics approval and consent to participate}

40 samples of HSCC and matched adjacent normal specimens were obtained from patients with HSCC who did not receive radiotherapy or chemotherapy at Third Xiangya hospital of Central South University hospital from January 2016 to June 2020 . All samples were stored in $-80^{\circ} \mathrm{C}$ until analysis. The study was approved by the Ethics Committee of Third Xiangya hospital of Central South University, and all subjects signed written informed consent.

All animal experiments were approved by the Animal Ethics Committee of Third Xiangya hospital of Central South University. 


\section{Consent for publication}

The informed consent obtained from study participants

\section{Availability of data and material}

All data generated or analysed during this study are included in this published article

\section{Competing interests}

The authors have no commercial or other associations that might pose a conflict of interest.

\section{Funding}

This work was supported by Hunan Youth Talents Project (No.2020).

\section{Authors' Contributions}

The authors have no commercial or other associations that might pose a conflict of interest.

\section{Acknowledgements}

We would like to give our sincere gratitude to the reviewers for their constructive comments.

\section{References}

1. Cooper JS, Porter K, Mallin K, Hoffman HT, Weber RS, Ang KK, Gay EG, Langer CJ (2009) National Cancer Database report on cancer of the head and neck: 10-year update. Head Neck. 31: 748-58. doi: 10.1002/hed.21022

2. Hock BD, McKenzie JL, Patton WN, Haring LF, Yang Y, Shen Y, Estey EH, Albitar M (2003) The clinical significance of soluble CD86 levels in patients with acute myeloid leukemia and myelodysplastic syndrome. Cancer. 98: 1681-8. doi: 10.1002/cncr.11693

3. Sewnaik A, Hoorweg JJ, Knegt PP, Wieringa MH, van der Beek JM, Kerrebijn JD (2005) Treatment of hypopharyngeal carcinoma: analysis of nationwide study in the Netherlands over a 10-year period. Clin Otolaryngol. 30: 52-7. doi: 10.1111/j.1365-2273.2004.00913.x

4. Tsou YA, Lin MH, Hua CH, Tseng HC, Chen SW, Yang SN, Liang JA, Tsai MH (2007) Survival outcome by early chemoradiation therapy salvage or early surgical salvage for the treatment of hypopharyngeal cancer. Otolaryngol Head Neck Surg. 137: 711-6. doi: 10.1016/j.otohns.2007.07.022

5. Wycliffe ND, Grover RS, Kim PD, Simental A, Jr. (2007) Hypopharyngeal cancer. Top Magn Reson Imaging. 18: 243-58. doi: 10.1097/RMR.0b013e3181570c3f

6. Topalian SL, Drake CG, Pardoll DM (2012) Targeting the PD-1/B7-H1(PD-L1) pathway to activate anti-tumor immunity. Curr Opin Immunol. 24: 207-12. doi: 10.1016/j.coi.2011.12.009 
7. Zou W, Chen L (2008) Inhibitory B7-family molecules in the tumour microenvironment. Nat Rev Immunol. 8: 467-77. doi: 10.1038/nri2326

8. Pardoll DM (2012) The blockade of immune checkpoints in cancer immunotherapy. Nat Rev Cancer. 12: $252-64$. doi: $10.1038 / n r c 3239$

9. Wintterle S, Schreiner B, Mitsdoerffer M, Schneider D, Chen L, Meyermann R, Weller M, Wiendl H (2003) Expression of the B7-related molecule B7-H1 by glioma cells: a potential mechanism of immune paralysis. Cancer Res. 63: 7462-7.

10. Hodgson A, Slodkowska E, Jungbluth A et al. (2018) PD-L1 Immunohistochemistry Assay Concordance in Urothelial Carcinoma of the Bladder and Hypopharyngeal Squamous Cell Carcinoma. Am J Surg Pathol. 42: 1059-66. doi: 10.1097/PAS.0000000000001084

11. Cui P, Jing P, Liu X, Xu W (2020) Prognostic Significance of PD-L1 Expression and Its Tumor-Intrinsic Functions in Hypopharyngeal Squamous Cell Carcinoma. Cancer Manag Res. 12: 5893-902. doi: 10.2147/CMAR.S257299

12. Desmet CJ, Gallenne T, Prieur A et al. (2013) Identification of a pharmacologically tractable Fra1/ADORA2B axis promoting breast cancer metastasis. Proc Natl Acad Sci U S A. 110: 5139-44. doi: $10.1073 /$ pnas. 1222085110

13. Xu L, Hu H, Zheng LS et al. (2020) ETV4 is a theranostic target in clear cell renal cell carcinoma that promotes metastasis by activating the pro-metastatic gene FOSL1 in a PI3K-AKT dependent manner. Cancer Lett. 482: 74-89. doi: 10.1016/j.canlet.2020.04.002

14. Cui YP, Xie M, Pan WX, Zhang ZY, Li WF (2020) HOXA10 promotes the development of bladder cancer through regulating FOSL1. Eur Rev Med Pharmacol Sci. 24: 2945-54. doi: 10.26355/eurrev_202003_20659

15. Chan JJ, Tay Y (2018) Noncoding RNA:RNA Regulatory Networks in Cancer. Int J Mol Sci. 19. doi: $10.3390 /$ ijms 19051310

16. Ren X (2020) Genome-wide analysis reveals the emerging roles of long non-coding RNAs in cancer. Oncol Lett. 19: 588-94. doi: 10.3892/ol.2019.11141

17. Qian Y, Liu D, Cao S, Tao Y, Wei D, Li W, Li G, Pan X, Lei D (2017) Upregulation of the long noncoding RNA UCA1 affects the proliferation, invasion, and survival of hypopharyngeal carcinoma. Mol Cancer. 16: 68. doi: 10.1186/s12943-017-0635-6

18. Huan L, Guo T, Wu Y, Xu L, Huang S, Xu Y, Liang L, He X (2020) Hypoxia induced LUCAT1/PTBP1 axis modulates cancer cell viability and chemotherapy response. Mol Cancer. 19: 11. doi: 10.1186/s12943-019-1122-z

19. Iwai Y, Ishida M, Tanaka Y, Okazaki T, Honjo T, Minato N (2002) Involvement of PD-L1 on tumor cells in the escape from host immune system and tumor immunotherapy by PD-L1 blockade. Proc Natl Acad Sci U S A. 99: 12293-7. doi: 10.1073/pnas.192461099

20. Elias MM, Hilgers FJ, Keus RB, Gregor RT, Hart AA, Balm AJ (1995) Carcinoma of the pyriform sinus: a retrospective analysis of treatment results over a 20-year period. Clin Otolaryngol Allied Sci. 20: 249-53. doi: 10.1111/j.1365-2273.1995.tb01860.x 
21. Wahlberg PC, Andersson KE, Biorklund AT, Moller TR (1998) Carcinoma of the hypopharynx: analysis of incidence and survival in Sweden over a 30-year period. Head Neck. 20: 714-9. doi:

10.1002/(sici)1097-0347(199812)20:8<714::aid-hed9>3.0.co;2-2

22. Prensner JR, Chinnaiyan AM (2011) The emergence of IncRNAs in cancer biology. Cancer Discov. 1: 391-407. doi: 10.1158/2159-8290.CD-11-0209

23. Gutschner T, Diederichs S (2012) The hallmarks of cancer: a long non-coding RNA point of view. RNA Biol. 9: 703-19. doi: 10.4161/rna.20481

24. Zhou J, Li W, Jin T, Xiang X, Li M, Wang J, Li G, Pan X, Lei D (2015) Gene microarray analysis of IncRNA and mRNA expression profiles in patients with hypopharyngeal squamous cell carcinoma. Int J Clin Exp Med. 8: 4862-82.

25. Zhou J, Li M, Yu W, Li W, Wang J, Xiang X, Li G, Pan X, Lei D (2016) AB209630, a long non-coding RNA decreased expression in hypopharyngeal squamous cell carcinoma, influences proliferation, invasion, metastasis, and survival. Oncotarget. 7: 14628-38. doi: 10.18632/oncotarget.7403

26. Pauken KE, Wherry EJ (2015) Overcoming T cell exhaustion in infection and cancer. Trends Immunol. 36: 265-76. doi: 10.1016/j.it.2015.02.008

27. Dermani FK, Samadi P, Rahmani G, Kohlan AK, Najafı R (2019) PD-1/PD-L1 immune checkpoint: Potential target for cancer therapy. J Cell Physiol. 234: 1313-25. doi: 10.1002/jcp.27172

28. Hu C, Tian S, Lin L, Zhang J, Ding H (2020) Prognostic and clinicopathological significance of PD-L1 and tumor infiltrating lymphocytes in hypopharyngeal squamous cell carcinoma. Oral Oncol. 102: 104560. doi: 10.1016/j.oraloncology.2019.104560

29. Zhao M, Sun D, Li X, Xu Y, Zhang H, Qin Y, Xia M (2017) Overexpression of long noncoding RNA PEG10 promotes proliferation, invasion and metastasis of hypopharyngeal squamous cell carcinoma. Oncol Lett. 14: 2919-25. doi: 10.3892/ol.2017.6498

30. Xu J, Bo Q, Zhang X, Lei D, Wang J, Pan X (2020) IncRNA HOXA11-AS Promotes Proliferation and Migration via Sponging miR-155 in Hypopharyngeal Squamous Cell Carcinoma. Oncol Res. 28: $311-$ 9. doi: $10.3727 / 096504020 \times 15801233454611$

31. Ashrafizadeh M, Zarrabi A, Hushmandi K, Zarrin V, Moghadam ER, Zabolian A, Tavakol S, Samarghandian S, Najafi M (2020) PD-1/PD-L1 axis regulation in cancer therapy: The role of long non-coding RNAs and microRNAs. Life Sci. 256: 117899. doi: 10.1016/j.Ifs.2020.117899

32. Mineo M, Lyons SM, Zdioruk M et al. (2020) Tumor Interferon Signaling Is Regulated by a IncRNA INCR1 Transcribed from the PD-L1 Locus. Mol Cell. 78: 1207-23 e8. doi: 10.1016/j.molcel.2020.05.015

33. Zhao L, Liu Y, Zhang J, Liu Y, Qi Q (2019) LncRNA SNHG14/miR-5590-3p/ZEB1 positive feedback loop promoted diffuse large $B$ cell lymphoma progression and immune evasion through regulating PD-1/PD-L1 checkpoint. Cell Death Dis. 10: 731. doi: 10.1038/s41419-019-1886-5

34. Jiang X, Xie H, Dou Y, Yuan J, Zeng D, Xiao S (2020) Expression and function of FRA1 protein in tumors. Mol Biol Rep. 47: 737-52. doi: 10.1007/s11033-019-05123-9 
35. Elangovan IM, Vaz M, Tamatam CR, Potteti HR, Reddy NM, Reddy SP (2018) FOSL1 Promotes Krasinduced Lung Cancer through Amphiregulin and Cell Survival Gene Regulation. Am J Respir Cell Mol Biol. 58: 625-35. doi: 10.1165/rcmb.2017-01640C

36. Luo YZ, He P, Qiu MX (2018) FOSL1 enhances growth and metastasis of human prostate cancer cells through epithelial mesenchymal transition pathway. Eur Rev Med Pharmacol Sci. 22: 8609-15. doi: 10.26355/eurrev_201812_16624

37. Knoch KP, Bergert H, Borgonovo B, Saeger HD, Altkruger A, Verkade P, Solimena M (2004) Polypyrimidine tract-binding protein promotes insulin secretory granule biogenesis. Nat Cell Biol. 6 : 207-14. doi: 10.1038/ncb1099

38. Sawicka K, Bushell M, Spriggs KA, Willis AE (2008) Polypyrimidine-tract-binding protein: a multifunctional RNA-binding protein. Biochem Soc Trans. 36: 641-7. doi: 10.1042/BST0360641

39. Li J, Yang Y, Fan J, Xu H, Fan L, Li H, Zhao RC (2019) Long noncoding RNA ANCR inhibits the differentiation of mesenchymal stem cells toward definitive endoderm by facilitating the association of PTBP1 with ID2. Cell Death Dis. 10: 492. doi: 10.1038/s41419-019-1738-3

\section{Figures}

A

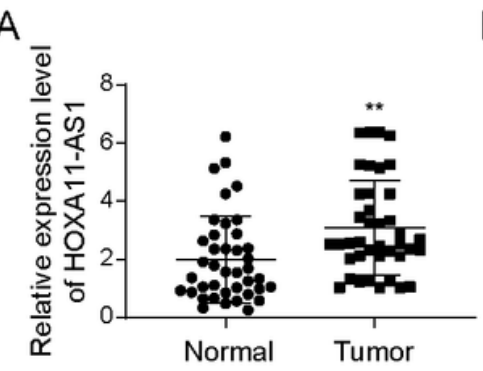

E

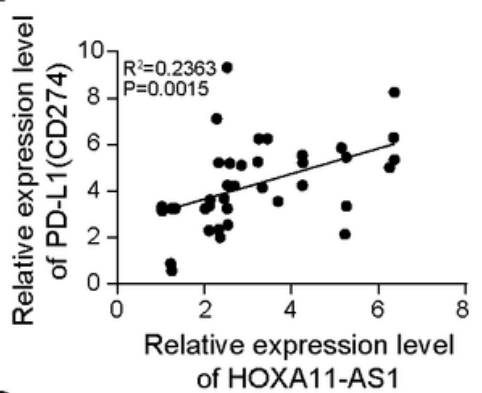

G

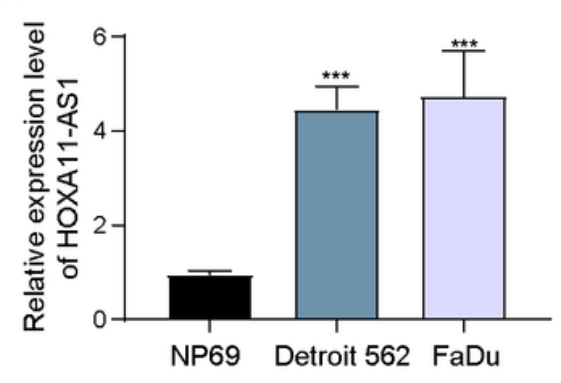

$\mathrm{B}$

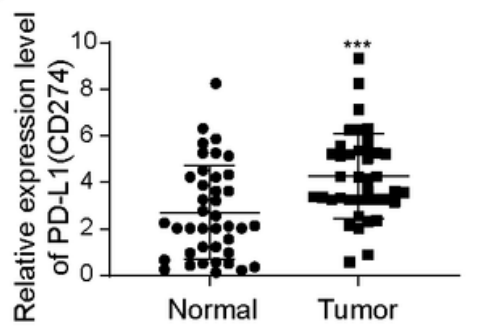

$\mathrm{F}$
C
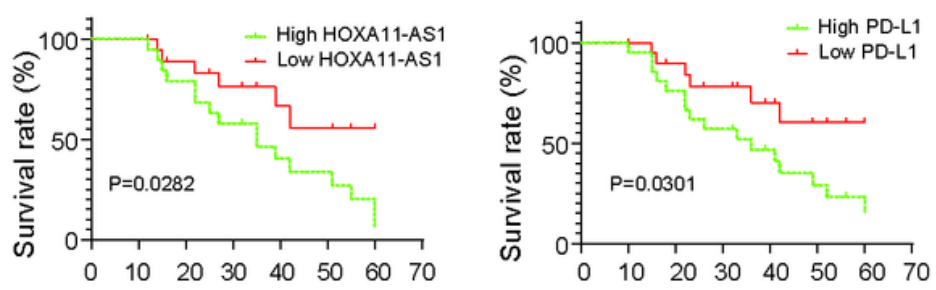

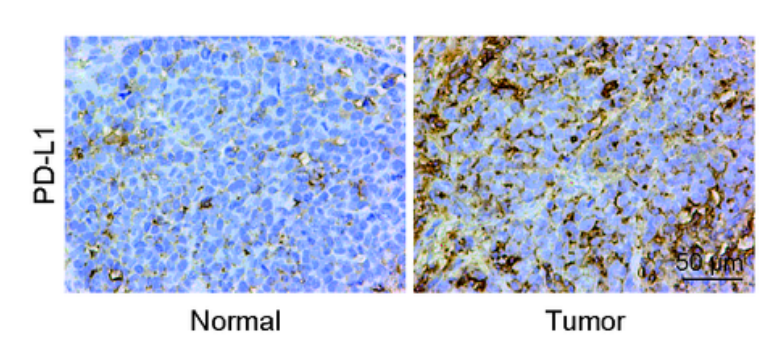

$\mathrm{H}$
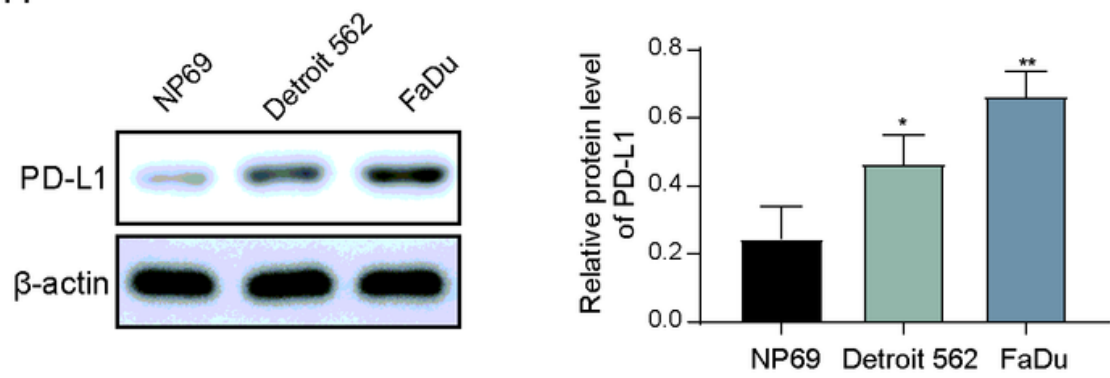

Figure 1 
HOXA11-AS1 and PD-L1 showed upregulation in HSCC, and HOXA11-AS1 was positively correlated with PD-L1. (A and B) HOXA11-AS1 and PD-L1 expression in 40 HSCC tissues and matched adjacent normal tissues were measured by RT-qPCR. (C and D) Kaplan-Meier overall survival curve stratified by HOXA11AS1 and PD-L1 expression. (E) The correlation between HOXA11-AS1 and PD-L1 expression was analyzed by Pearson's correlation coefficient. (F) PD-L1 expression in HSCC tissues was measured by immunohistochemical staining. ( $(\mathbf{a}$ and $\mathbf{H}$ ) The mRNA expression of HOXA11-AS1 and the protein level of PD-L1 in NP69 and HSCC cells (FaDu and Detroit 562 cells) were detected by RT-qPCR and western blot, respectively. ${ }^{\star} P<0.05,{ }^{*} P<0.01,{ }^{\star \star *} P<0.001$. 
A

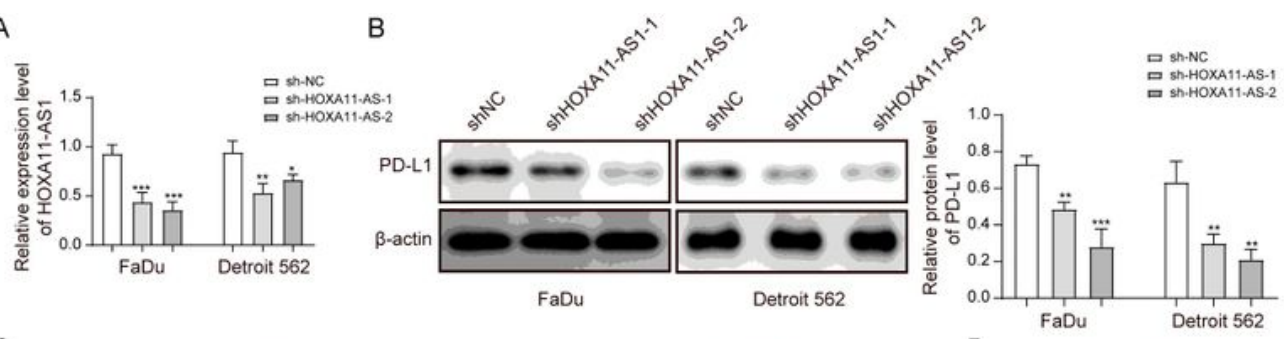

C
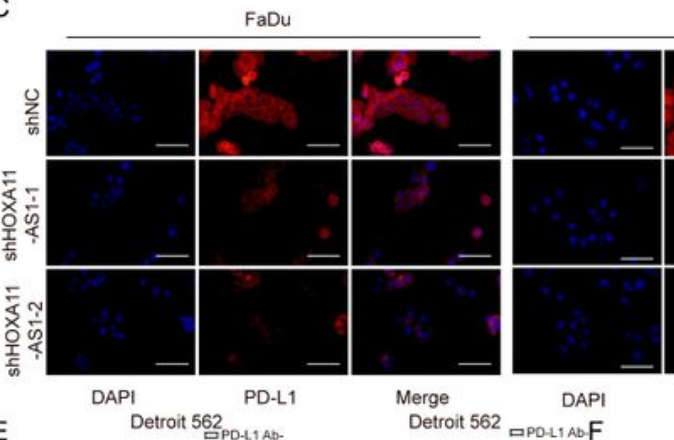

Detroit 562

$\mathrm{D}$

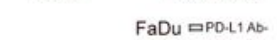

E

Detroit 562 PD-L1AB-

Merge

DAPI
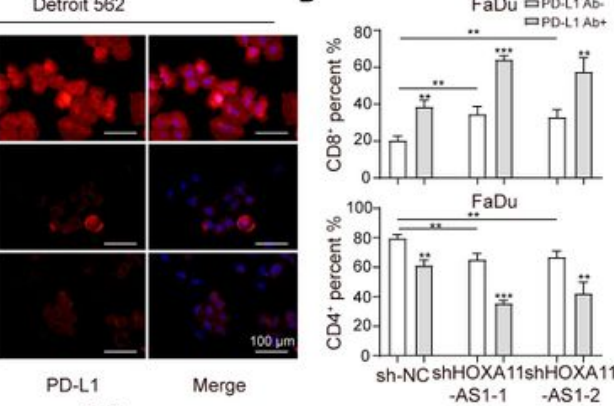

PD-L1 Merge
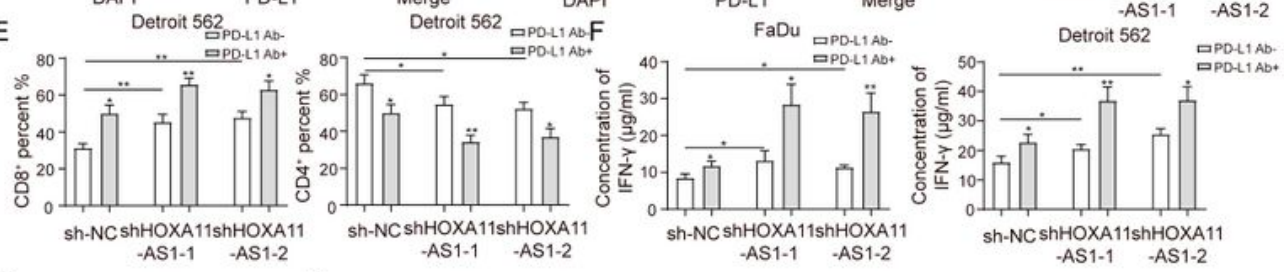

G

FaDu

$\mathrm{H}$

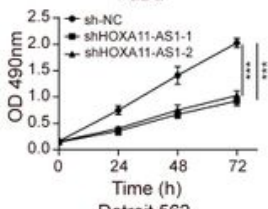

$2.0 \div$ Dh-NC 562

E1.5- ShHOXA11-AS1-1
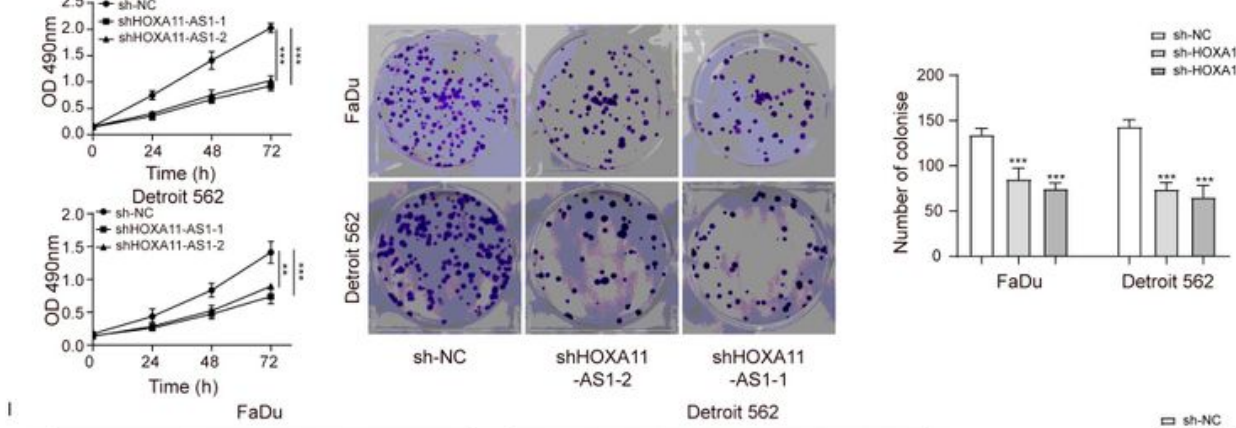

sh-NC

ShHOXA11

ShHOXA11

Detroit 562

므 Sh-NC

口ㄴ. Sh-HOXA11.AS-1
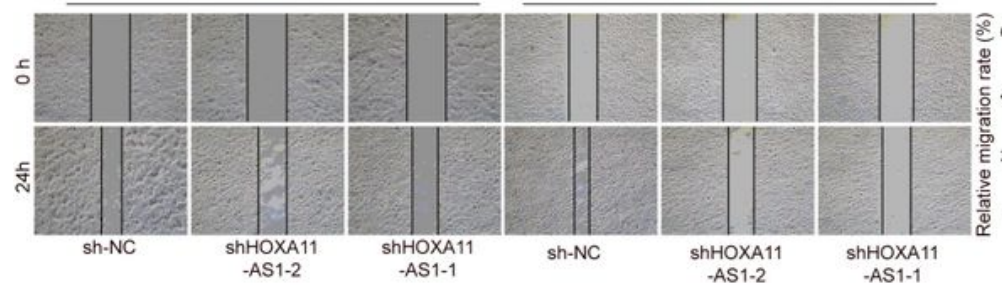

$\begin{array}{cc}\text { shHOXA11 } & \text { ShHOXA1 } \\ \text {-AS1-2 } & \text {-AS1-1 }\end{array}$

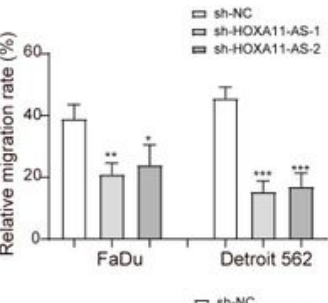

므 sh.NC

D. sh-HOXA11-AS-1
- sh-HOXA11-AS-2

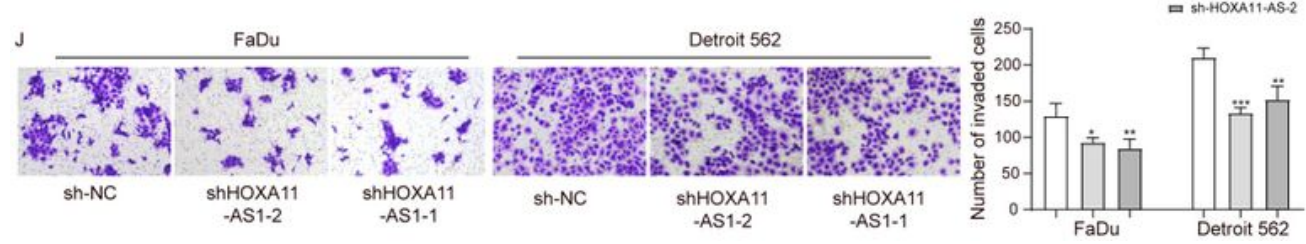

Figure 2

Knockdown of HOXA11-AS1 suppressed the expression of PD-L1, as well as the immune escape, proliferation and metastasis of HSCC cells. FaDu and Detroit cells were transfected with two specific shRNAs: shHOXA11-AS1-1 and shHOXA11-AS1-2. (A) The transfection efficiencies were measured by RTqPCR. (B and C) PD-L1 levels were detected after silencing of HOXA11-AS1 by western blot and immunofluorescence. Anti-PD-L1 antibodies restored the cytotoxic effect of T lymphocytes. Treated FaDu 
and Detroit cells were pretreated with or without anti-PD-L1 antibodies for $1 \mathrm{~h}$ and co-cultured with PBMC for $72 \mathrm{~h}$, then (D) the percentage of $C D 8^{+}$and $(E) C D 4^{+} T$ cells was analyzed by flow cytometry, and $(F)$ the concentration of IFN- $\gamma$ was measured by ELISA. (G-J) The viability, colony formation, migration and invasion of FaDu and Detroit cells were measured by CCK-8, colony formation, wound healing and transwell assays after knockdown of HOXA11-AS1. ${ }^{*} P<0.05,{ }^{\star} P<0.01, * \star \star P<0.001$.
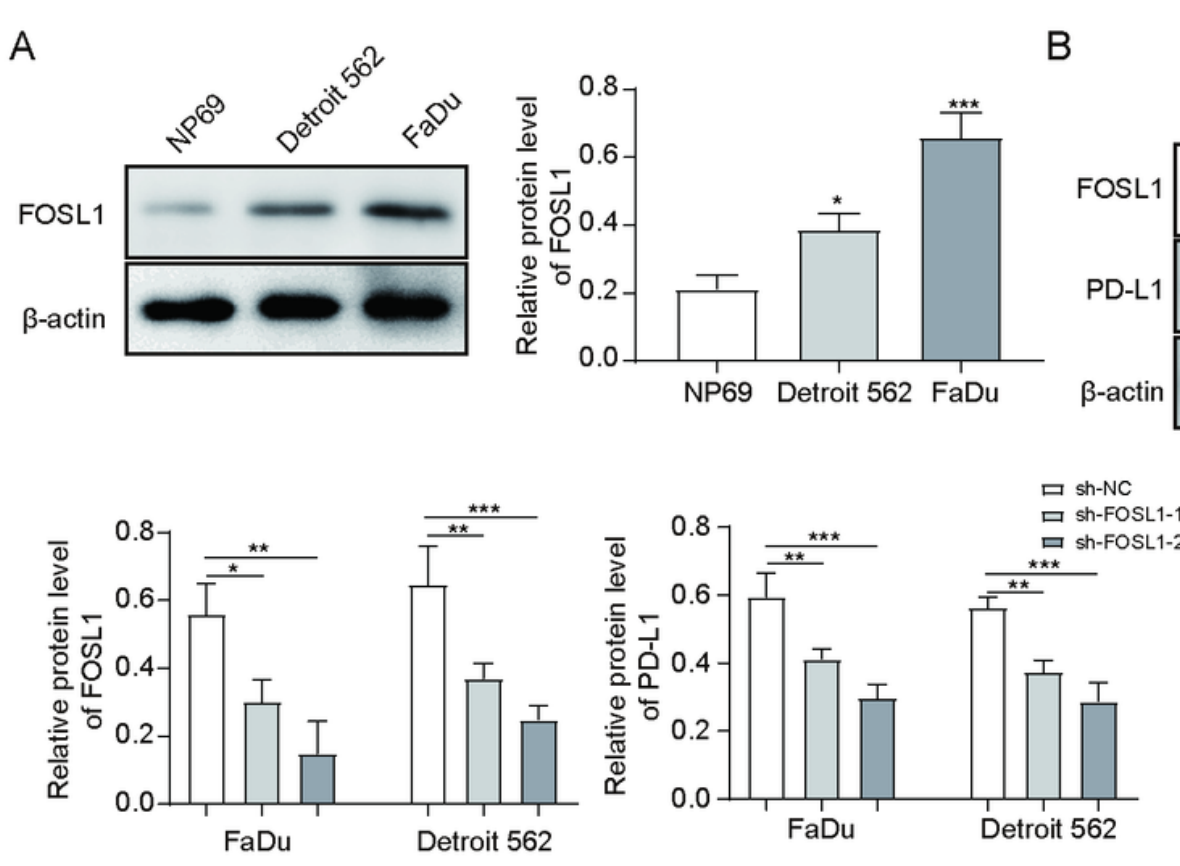

$C$
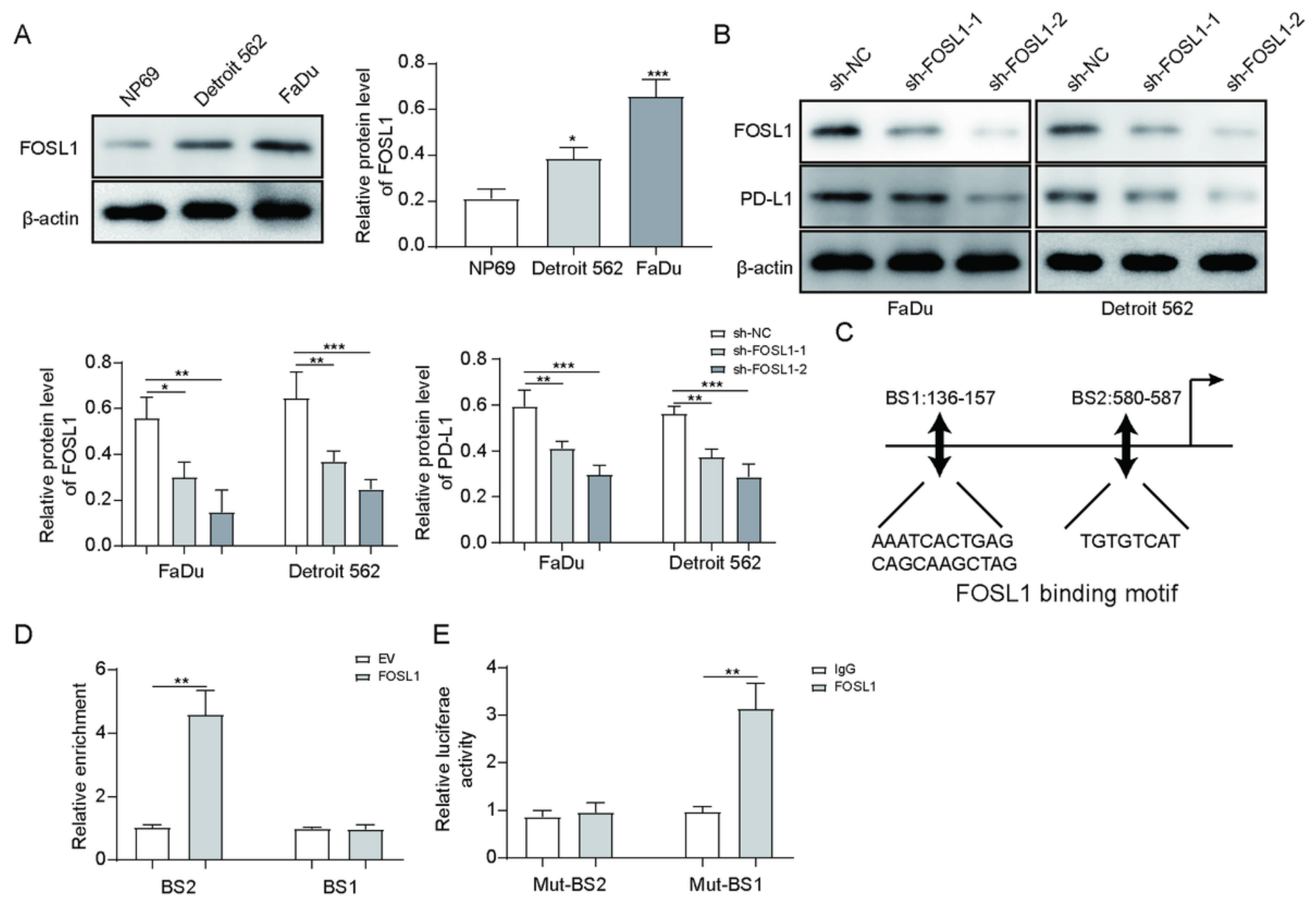

E

\section{Figure 3}

FOSL1 was highly expressed in HSCC cells and positively regulated PD-L1 expression through binding to the promoter of PD-L1. (A) The protein level of FOSL1 in HSCC cells was measured by western blot. (B) FaDu and Detroit 562 cells were transfected with shFOSL1-1 and shFOSL1-2, then the expression of FOSL1 and PD-L1 was detected by western blot. (C) JASPAR was used to predict the binding sites between FOSL1 and the promoter of PD-L1. (D and E) ChIP and dual-luciferase reporter assays were used to further validate the regulatory relationship between FOSL1 and the promoter of PD-L1. ${ }^{\star} P<0.05,{ }^{*} P<$ $0.01,{ }^{*} * * 0.001$. 
A

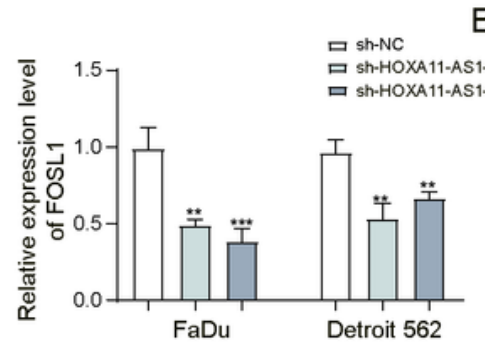

C

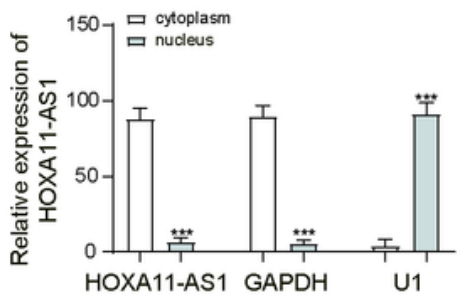

$B$

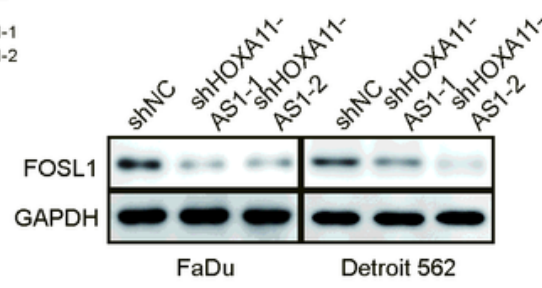

D
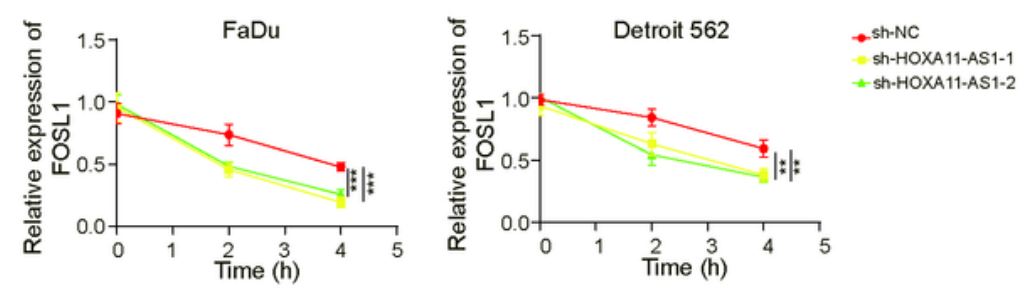

E

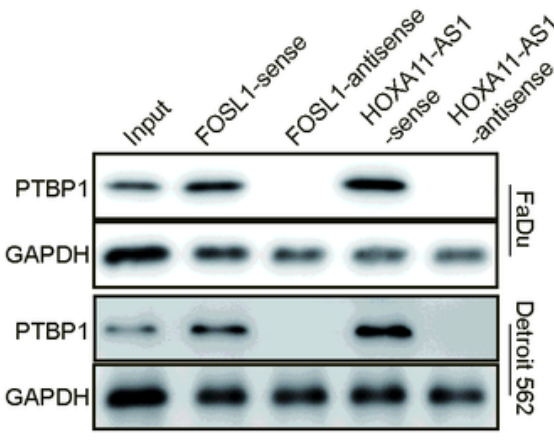

F
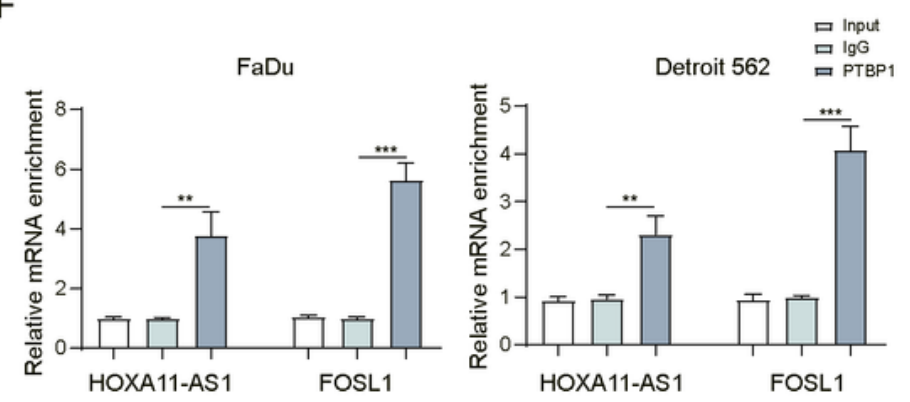

G

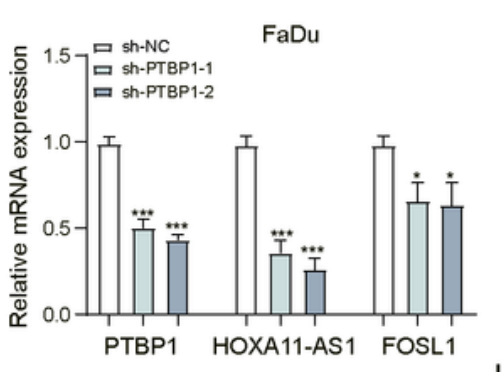

Detroit 562
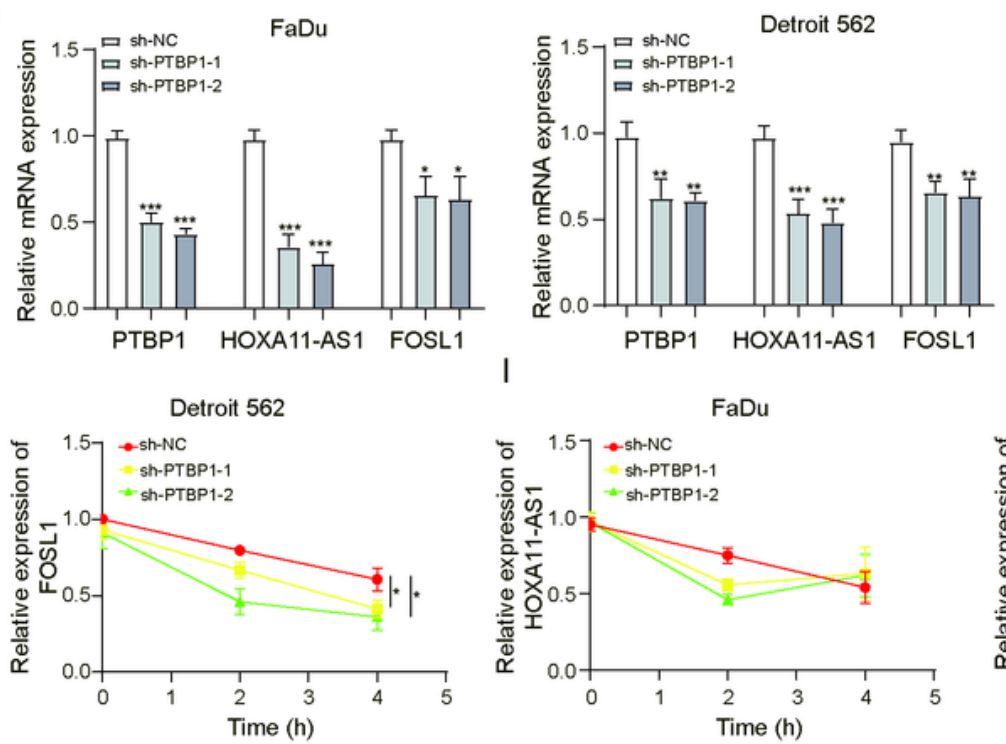

$\mathrm{H}$

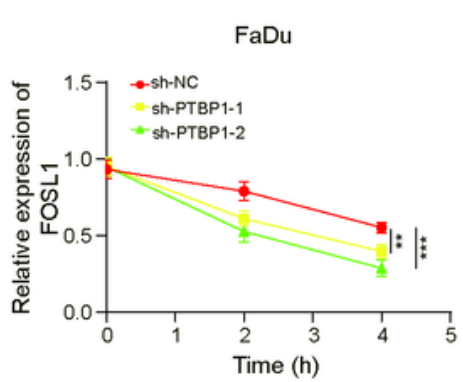

FaDu
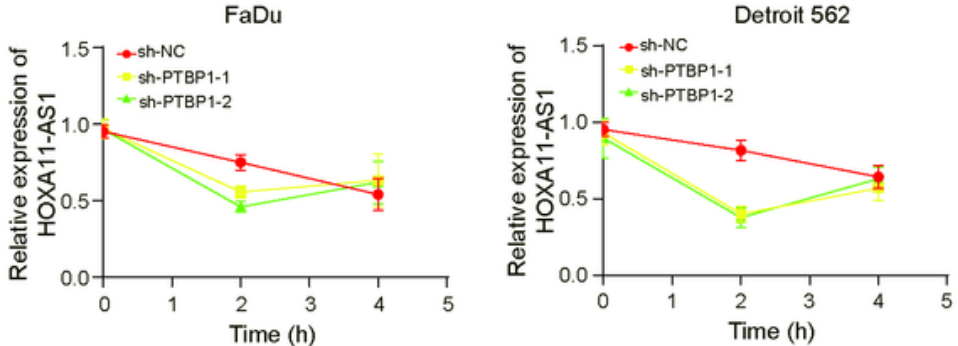

\section{Figure 4}

HOXA11-AS1 enhanced the stability of FOSL1 mRNA through combining with PTBP1. (A and B) FOSL1 levels were evaluated using RT-qPCR and western blot after knockdown of HOXA11-AS1, respectively. (C) The cytoplasm and nucleus of FaDU cells were collected to measure the fraction of HOXA11-AS1. (D) FOSL1 mRNA decay in FaDu and Detroit cells was measured after treating with actinomycin D (ACD) after knockdown of HOXA11-AS1. (E) The protein analysis of PTBP1 after RNA pull down with biotin- 
labelled sense or antisense HOXA11-AS1 or FOSL1. (F) RIP assay was performed to measure the binding abundance of PTBP1 and FOSL1 mRNA or HOXA11-AS1. FaDu and Detroit cells were transfected with shPTBP1, then (G) PTBP1, FOSL1 and HOXA11-AS1 levels were evaluated through RT-qPCR, and (H) FOSL1 and (I) HOXA11-AS1 mRNA decay was measured after ACD treatment. ${ }^{\star} P<0.05,{ }^{\star *} P<0.01, * \star \star P<0.001$.
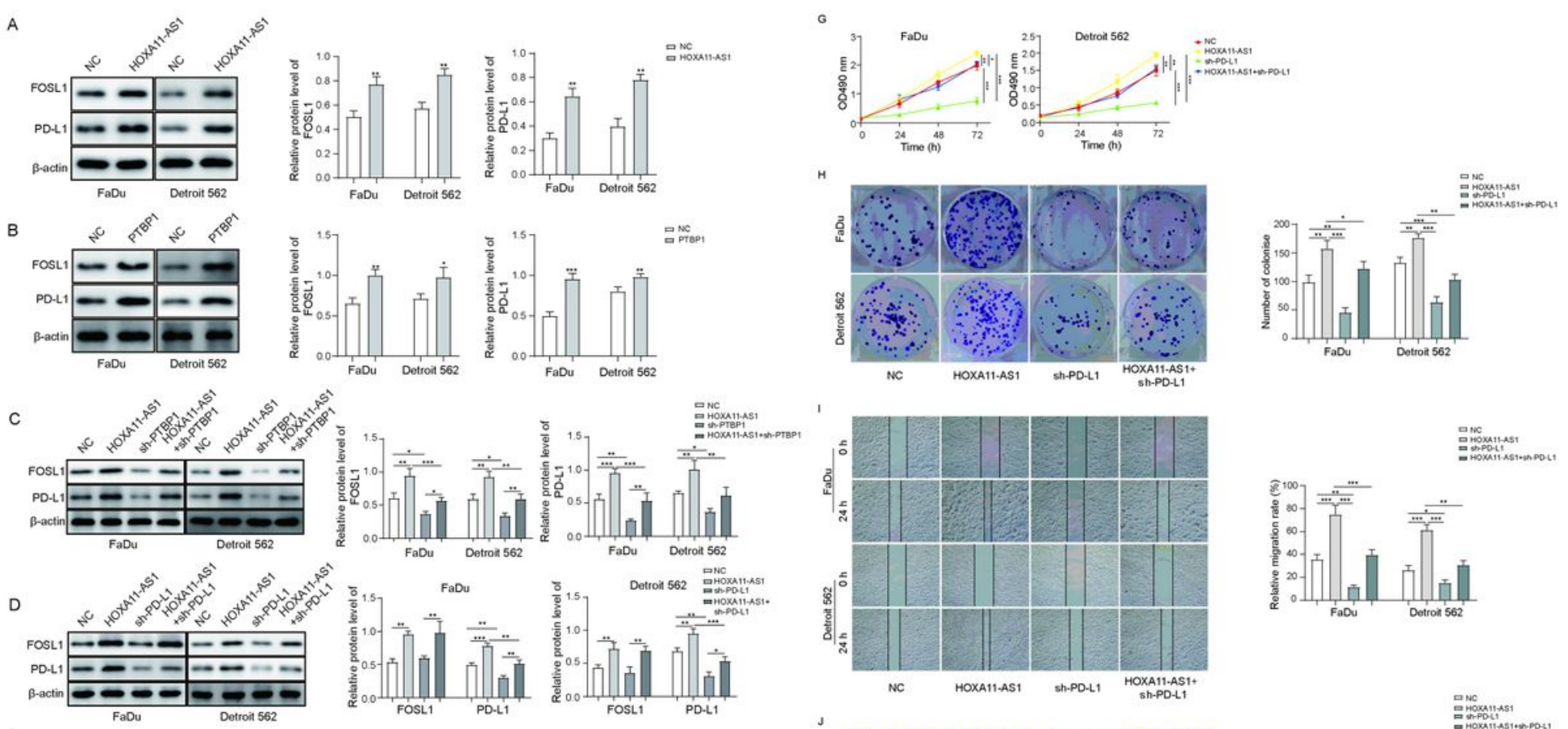

E
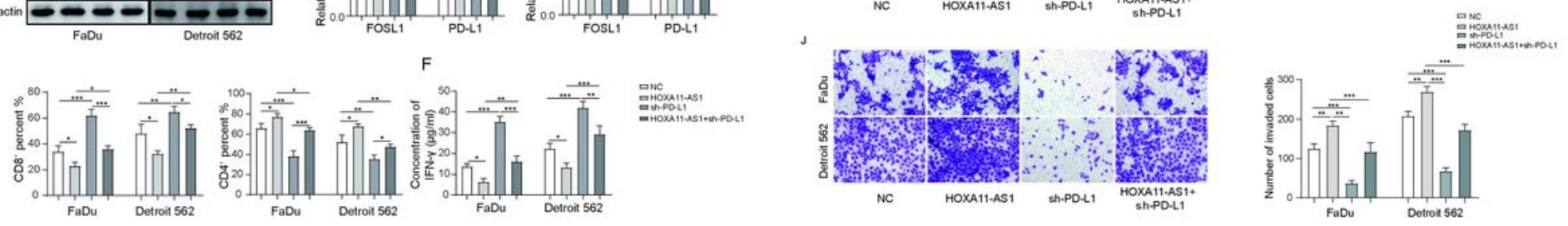

Figure 5

HOXA11-AS1 promoted PD-L1 expression through up-regulating FOSL1 levels by PTBP1, thereby facilitating the immune escape, growth and metastasis of HSCC cells. FaDu and Detroit cells were transfected with pcDNA3.1-HOXA11-AS1 (HOXA11-AS1), pcDNA3.1-PTBP1 (PTBP1), shPTBP1 or the combination of HOXA11-AS1+shPTBP1. (A and B) FOSL1 and PD-L1 levels were measured by western blot after overexpression of HOXA11-AS1 or PTBP1. (C) Western blot was used to measure FOSL1 and PD-L1 levels in HOXA11-AS1, shPTBP1 or HOXA11-AS1+shPTBP1 groups. FaDu and Detroit cells were transfected with HOXA11-AS1, shPD-L1 or the combination of HOXA11-AS1+shPD-L1, (D) Western blot was used to measure FOSL1 and PD-L1 levels, then (E) the percentage of $C D 8^{+}$and $C D 4^{+} T$ cells and $(F)$ the concentration of IFN-y were measured by flow cytometry and ELISA, respectively, and (G-J) the viability, colony formation, migration and invasion of treated HSCC cells were analyzed by CCK-8, colony formation, wound healing and transwell assays. ${ }^{*} P<0.05,{ }^{\star} P<0.01, \star \star \star P<0.001$. 
A
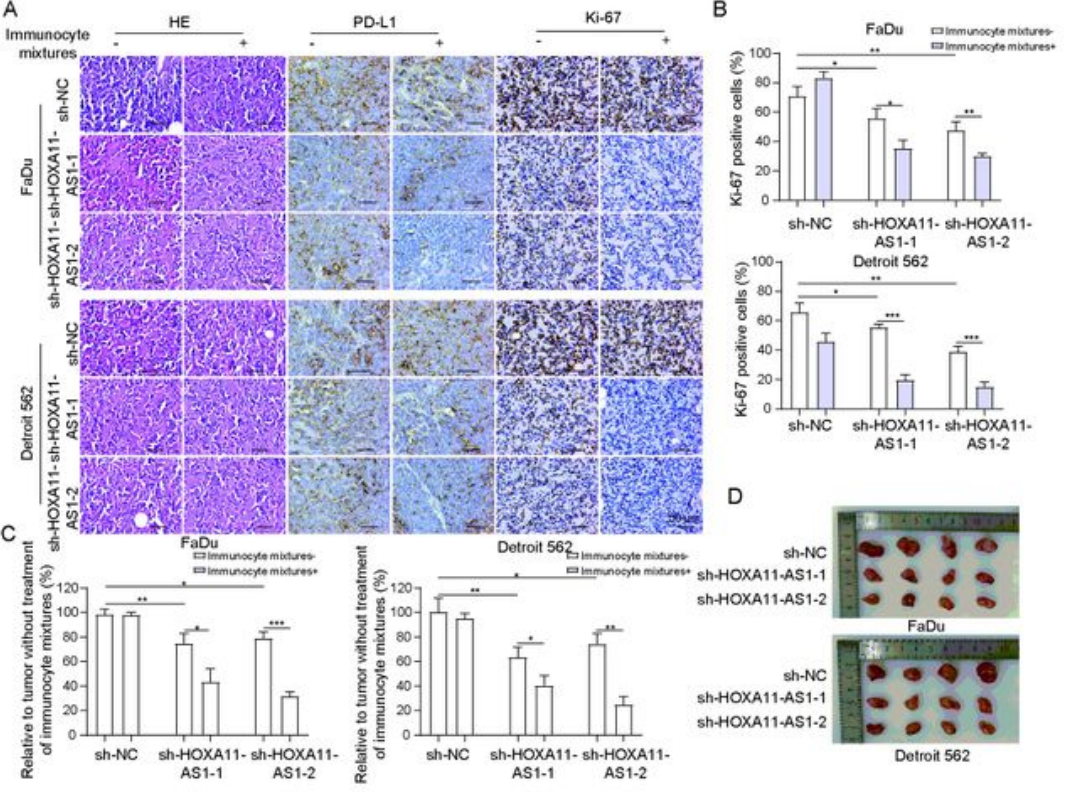

E
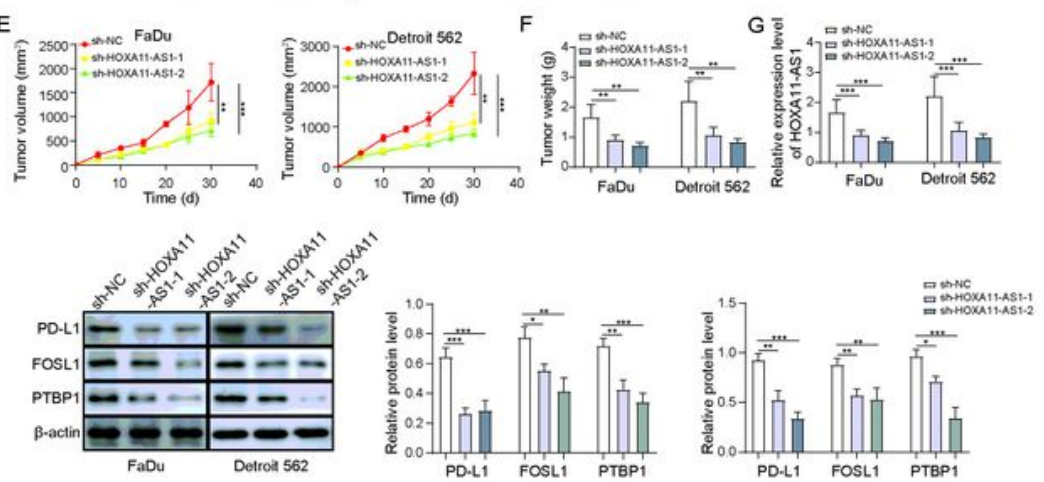

$\mathrm{H}$
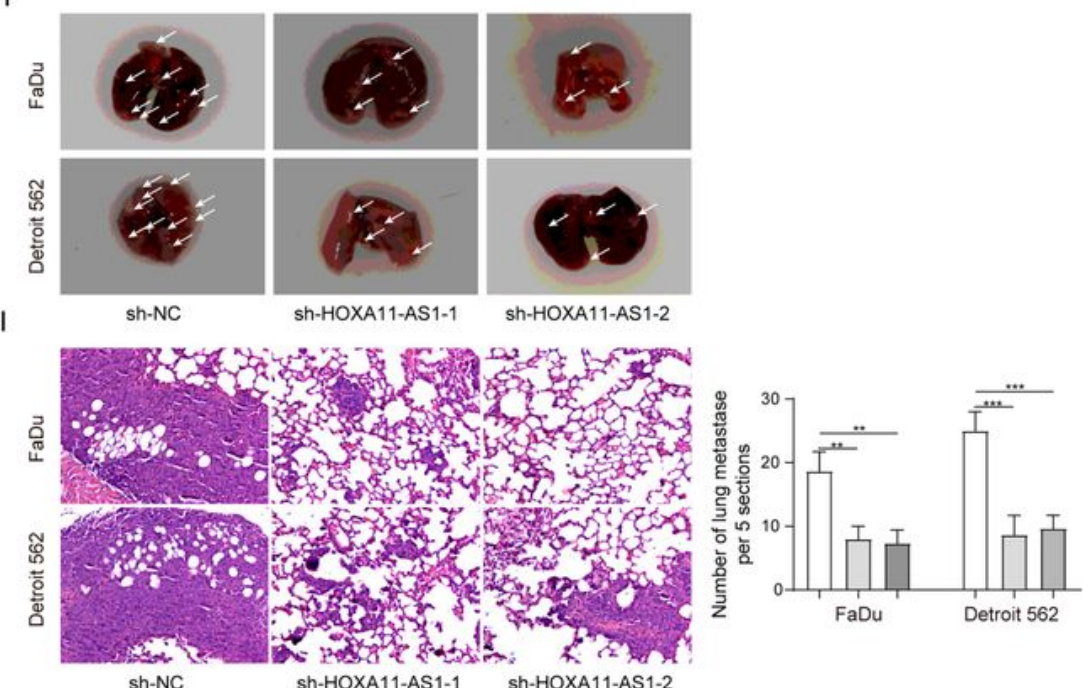

sh-NC

sh-HOXA11-AS1-

sh-HOXA11-AS1-2

\section{Figure 6}

knockdown of HOXA11-AS1 inhibited the immune escape and metastasis through regulating PD-L1 by down-regulating FOSL1 in vivo. FaDu and Detroit cells stably expressed shNC, shHOXA11-AS1-1 or shHOXA11-AS1-2 were injected subcutaneously into NOD-SCID mice to establish xenograft models. PBS or PBMC was intraperitoneally injected into NOD-SCID mice to kill xenografts more effectively. (A) HE, Ki67 and IHC staining were used to measure tumor pathology, proliferation and PD-L1 expression of 
xenografts treated with/without PBMC or PBC. (B) The percentage of Ki67 cells were evaluated in xenografts. (C) The cytotoxic effect of the treatment with/without PBMC or PBS was measured by calculating xenograft volumes in shNC, shHOXA11-AS1-1, shHOXA11-AS1-2 xenografts, the results were shown in the bar chart as tumor regression rate. (D-F) tumor volume and weight were detected after knockdown of HOXA11-AS1 in vivo. (G) RT-qPCR was used to HOXA11-AS levels and western blot was used to measure the expression of PD-L1, FOSL1 and PTBP1 after knockdown of HOXA11-AS1. (H-I) The ability of metastasis to lung, tumor pathology and nodules number of lung metastases were measured

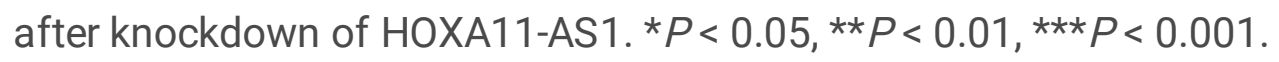

